\title{
Multi-Ensayos
}

Vol. 5, $\mathrm{N}^{\circ} 9$

ISSN: 2412-3285

https://multiensayos.unan.edu.ni

DOI: https://doi.org/10.5377/multiensayos.v5i9.9434

\section{La investigación de mercado una oportunidad para el aprendizaje en Mercadotecnia}

\section{Market research an opportunity for marketing learning}

Yasmina Ramírez Sobalvarro ${ }^{1}$

\section{RESUMEN}

La investigación de mercado es una de las áreas más abiertas y sujetas a cambios dentro de la Mercadotecnia. En la carrera que se desarrolla en la Facultad Regional Multidisciplinaria de Estelí, está asignatura se lleva durante un año completo distribuido en investigación de mercado I y II. En este momento este escrito presenta la experiencia desarrollada durante con IV año de la carrera de Mercadotecnia, curso durante el cual se evidenció la creatividad, compromiso, responsabilidad y deseo de aprender de los estudiantes. Igualmente la motivación y la disciplina que representó en su momento la lectura de textos así como de documentos complementarios esto con la finalidad de acompañar los procesos teóricos con los prácticos, validándose de esta manera una mejor forma de aprender en las aulas de clase.

Palabras claves: investigación de mercado; experiencia metodológica.

\begin{abstract}
Market research is one of the most open areas and subject to changes in marketing. In the career that takes place in the Multidisciplinary Regional Faculty of Estelí, this subject is carried out for a year distributed in market research I and II. At this time this writing presents the experience developed during the IV year of the marketing career, in which creativity, commitment, and responsibility and wish to learn from the students was evidenced. Also, the motivation and the discipline that represented at the time the reading texts as well as complementary documents this with the purpose of accompanying the theoretical processes with the practical ones, validating in this way a better method to learn in the class.
\end{abstract}

Keywords: market research; methodological experience.

1 Docente del departamento de Ciencias Económicas y Administrativas. UNAN-Managua/FAREM-Estelí. Correo electrónico: jazminara72@gmail.com,yramirez@unan.edi.ni.

(C) 2019 Revista Multi-Ensayos. 


\section{INTRODUCCIÓN}

La investigación de mercado es la reunión, el registro y el análisis de todos los hechos acerca de los problemas relacionados con las actividades de las personas, las empresas y las instituciones en general. En el caso concreto de las empresas privadas, la investigación de mercados ayuda a la dirección a comprender su ambiente, identificar problemas y oportunidades, además de evaluar y desarrollar alternativas de acción de marketing. En el caso de las organizaciones públicas, la investigación de mercados contribuye a una mejor comprensión del entorno que les permite tomar mejores decisiones de tipo económico, político y social. (Benassini, 2009)

Como parte fundamental de la Investigación de Mercados se encuentra el hecho de que es una de las aristas más valiosas para el desarrollo de la carrera de Mercadotecnia, y le brinda la oportunidad a los estudiantes de realizar procesos de investigación con carácter profesional.

De hecho en la actualidad la investigación de mercado es vista como una herramienta por parte de las empresas a nivel nacional, las cuales con el tiempo se han dado cuenta de la importancia de conocer sobre los gustos, preferencias y aspectos relacionados con las tendencias que se marcan.

La investigación de mercado al no ser tan difundida su uso dentro del país, su realización representa un alto costo, pero al mismo tiempo la plusvalía que genera la misma bien encauzada y realizada por profesionales es casi una garantía de que se obtendrán oportunidades e información oportuna para la toma de decisiones inteligentes.

\section{DESARROLLO}

La investigación de mercados nació cuando surge el comercio. Informalmente el oferente hacía indagaciones para saber que quería la gente y que hacían sus competidores. La investigación de mercados como ciencia nació en la segunda mitad del siglo XIX cuando se empezaron a aplicar cuestionarios.

En 1911 Charles Carlin de Curtis Publishing Group funda el primer departamento de investigación de mercados. Carlin es celebre porque esculcó la basura de la gente para demostrarle a Campbell's Soup que debía pautar en uno de sus diarios. Entre los años 20's y 40's se populariza el uso de encuestas para conocer preferencias del consumidor.

Después de la segunda guerra mundial la gente de mercadeo incorpora las recién desarrolladas técnicas de muestreo estadístico a la investigación de mercados. Nace la investigación cuantitativa. Al mismo tiempo un equipo de sicólogos experimenta con grupos de personas, bajo la hipótesis de que la reacción a una serie de estímulos permitirá obtener información de hábitos, expectativas, etc. Nace las investigación cualitativa a finales de los 40's. El boom económico de posguerra favorece el desarrollo de la investigación de mercados. Paralelo al crecimiento de la producción y ventas de bienes: autos, televisores, neveras y lavadoras, crecen los negocios de la publicidad y de la investigación de mercados.

En los 80's la orientación a la producción y a las ventas cede paso a la orientación al consumidor. Toma fuerza la investigación cualitativa sin desplazar a la cuantitativa. A finales de los 90's convergen la intensificación de la competencia que genera variedad de marcas y productos y la masificación de internet. 
Entonces el consumidor cambia: es más difícil conocerlo y venderle. Surgen desafíos y oportunidades para la gente de mercadeo: El desafío: conocer mejor al consumidor y anticiparse con menor error a sus decisiones. La oportunidad: la disponibilidad de un canal sincrónico, barato y de alcance global. Nace la e-survey y la chatnography - etnografía de chatrooms. También surgen oportunidades en la recolección, transmisión y análisis de datos. Más información, más rápido...Y aquí estamos, parados frente al futuro.

\section{El futuro de la investigación de mercados}

El futuro de la investigación de mercados está siendo moldeado por tres fuerzas muy importantes:

1. Poder del consumidor. Hoy y en el futuro, el consumidor tiene la libertad de escoger y cambiar cuando quiera. Es él quien decide. La sobreoferta de productos, productores y marcas además de la oferta de información, le permiten al consumidor escoger y decidir autónomamente en la mayoría de mercados de bienes y servicios de consumo masivo.

Bajo estas circunstancias, las empresas productoras de bienes y servicios deberán conocer mejor y de manera permanente a los consumidores. Les exigirán a las investigadoras de mercados más iniciativa y celeridad a la hora de desenmascarar al consumidor.

2. Intensificación de la competencia. Las manufactureras y los retailers ${ }^{1}$ constituidas han visto y verán como emergen dinámicamente nuevos competidores particularmente en China y otros países neo industrializados. Proliferan marcas y genéricos, seguidores y copiones que amenazan la rentabilidad de los negocios. El desafío es atender mejor al consumidor por medio de una mejor oferta de productos y servicios. Como atenderlo mejor? Aplicando el principio fundamental del mercadeo: conocer y entender al consumidor para anticiparse a sus expectativas mediante la oferta de productos y servicios que las superen. (Altamirano López, 2010)

Para los investigadores de mercadeo de nuevo el desafío es conocer mejor al consumidor y reducir los ciclos de "estudio" mejor dicho producir más información de mejor calidad más rápido.

3. Avance de las tecnologías de recolección, transmisión y organización de datos. Avance que hoy nos permite hacer in-house e in-store data scanning ${ }^{2}$ para tener en tiempo casi real reportes comparados del comportamiento de productos, marcas y categorías.

Esta fuerza les exigirá a los investigadores de mercados mayor capacidad para relacionar e interpretar mayores volúmenes de información en el menor tiempo posible. Además los volúmenes de datos que nos permiten describir el comportamiento del consumidor deberán ser complementados con información más profunda de sus motivos y actitudes: sus porqués.

Como parte de la experiencia del año lectivo 2017 se desarrolló la estrategia de que cada estudiante pudiera presentar de manera individual un tema de investigación de mercado para aplicar los conocimientos adquiridos, la idea era fundamentalmente lograr que los estudiantes trabajaran de manera independiente, demostrando sus capacidades, habilidades y destrezas en cuanto a la elaboración de instrumentos, aplicación de los mismos en campo y por supuesto lo relacionado con la utilización de programas estadísticos, propios para el análisis de estos temas:

1 Ventas al por menor

2 Escaneo de datos internos en la tienda 
Dentro del stop de líneas para investigar figuran los relacionados con:

- Comportamiento de compra

- Compras compulsivas

- Merchandising

- Psicología del color

- Influencia de la publicidad entre otras.

Los estudiantes han tenido la oportunidad de interactuar con lecturas formativas, hacer análisis de las mismas, presentar sus opiniones sobre contenidos temáticos y además aplicar los conocimientos básicos y necesarios para la realización de una investigación de mercado en pequeño a negocios de la ciudad de Estelí.

Realmente son muchos los aprendizajes que esta experiencia ha suscitado entre las más particulares se encuentran:

- La disciplina de los estudiantes es fundamental para alcanzar el éxito

- El seguimiento vía correo electrónico

- Las consultas de manera semanal y sobre todo la combinación de los procesos teóricos y prácticos.

Se realizó un compartir de nuevas temáticas que enriquecen los conocimientos teóricos, algunas de estas se encuentran descritas a continuación:

- Personalidad y estilos de vida

- Los consumidores como individuos

- Las reglas de los consumidores

- El aprendizaje y la memoria

- La motivación y los valores

- Los consumidores en la toma de decisiones

Sobre estas temáticas se realizaron una serie de aplicaciones técnicas tales como:

- Debates

- Panel de expertos

- Programa de televisión entre otras formas activas de compartir el conocimiento

Igualmente se trabajó todo un portafolio de tareas donde los estudiantes tenían que emitir sus criterios para posteriormente interiorizar los nuevos retos que como Mercadotecnista se tienen que enfrentar. Es de vital importancia que los estudiantes logren precisar sobre este tipo de conocimientos puesto que se requiere que los mismos puedan asimilar y tener claro la forma de aplicarlos en la práctica.

Entre los propósitos centrales de esta asignatura se encuentran:

- Analizar la importancia del estudio del comportamiento del consumidor y las decisiones estratégicas de Mercadeo.

- Comprender de qué manera los factores personales, psicológicos, sociales y situacionales pueden afectar el proceso de decisión de compra del consumidor. 
Entre los conceptos más debatidos se encuentran: comportamiento del consumidor cubre muchas áreas: es el estudio de los procesos que intervienen cuando una persona o grupo selecciona, compra, usa o desecha productos, servicios, ideas o experiencias para satisfacer necesidades y deseos. (Malhotra K, 2012)

Algunas de las investigaciones realizadas como producto de esta experiencia han sido las siguientes:

- Factores que influyen en la decisión de compra de café, en el segundo semestre del año 2017 en la ciudad de Estelí.

- Influencia del diseño del producto en la conducta de compra del consumidor

- Influencia del Branding Emocional en la fidelización de clientes de Coca-Cola

- el benchmarking como una estrategia de mejora para la empresa BAC- CREDOMATIC

- Efectos de la publicidad en el desarrollo de conductas agresivas en niños y adultos. (10 a 24 años).

- Oferta y demanda del mercado de servicio Hotelero de Hotel Hex and Convention Center de la ciudad de Estelí

- Comportamiento de las personas respecto al consumo de las bebidas energéticas en la ciudad de Estelí

- Tendencias en la compra de productos de la marca ADIDAS

\section{CONCLUSIÓN}

Cuando se habla de Investigación de mercado se tiene que evidenciar que es más que una oportunidad para los estudiantes de la carrera puesto que no solamente pueden estar en contacto con el público, sino que además interactúan con el consumidor final y sus intermediarios dependiendo del proceso de la misma. Realmente durante este semestre fue un reto grande para la docente, puesto que el seguimiento y la calidad en la atención tenían que hacerse utilizando diferentes recursos TIC. Sin embargo los resultados al final de este curso representaron un 95\% de aprobación y una media de $75 \%$ en un grupo de 41 estudiantes.

Los estudiantes logran mayores y mejores resultados al trabajar utilizando metodologías activas que les permita el contacto con la fuente primaria de información (clientes- consumidores)

El establecimiento de líneas y temas de interés dentro de la asignatura permite centrarse no solo en temáticas actuales, sino que logran aportar al conocimiento teórico- práctico de los estudiantes en la carrera.

Es de vital importancia la realización de un seguimiento de calidad durante el transcurso de la materia, ya sea de manera presencial así como virtual puesto que de esta forma los estudiantes avanzan en su proceso de investigación y se construyen mecanismos que evidencian el trabajo que cada uno de ellos ha logrado a lo largo del curso.

Esta experiencia de que los estudiantes trabajen de manera individual, representa un plus esfuerzo para el docente, pero al final se siente una gran satisfacción porque se evalúa el proceso de una forma más concienzuda y los estudiantes van asumiendo su responsabilidad sin tener que delegarla en otros que es lo que muy frecuentemente pasa cuando se asigna procesos de investigación grupal. 


\section{REFERENCIAS}

Altamirano López, A. (2010). Qué son, para qué sirven y cómo se hacen las investigaciones de mercado? México: Continental. Recuperado el 20 de noviembre de 2017

Benassini, M. (2009). Introducción a la Investigación de Mercado. México, DF, México: Pearson Publicación.

Malhotra K, N. (2012). Investigación de mercados. México: Pearson. Recuperado el 18 de noviembre de 2017

Mejicanos, M. (10 de mayo de 2016). Academia.edu. Obtenido de Academia.edu: https://www. academia.edu/6131829/Nuevas_Tendencias_en_la_Investigacion_de_Mercado

Muñiz, R. (12 de septiembre de 2016). Marketing- XXI.com. Obtenido de Marketing- XXI.com: www. marketing-xxi.com/proceso de la investigación.24.htm

www.consumershead.com/mrhistory.htm. (15 de abril de 2014). Mercadeo y Publicidad.com. Obtenido de Mercadeo y Publicidad.com : www.consumershead.com/mrhistory.htm 